\title{
ANALYTICAL AND NUMERICAL MODELLING OF DEFLECTION OF CIRCULAR THREE-LAYER PIEZOELECTRIC TRANSDUCER
}

\author{
Grzegorz Mieczkowski, Dariusz Szpica, Andrzej Borawski \\ Bialystok University of Technology, Poland \\ g.mieczkowski@pb.edu.pl,d.szpica@pb.edu.pl, a.borawski@pb.edu.pl
}

\begin{abstract}
The paper deals with modelling of a circular piezoelectric actuator under electrical loading. A threelayer transducer is analysed. The outer layers of the transducer are made of non-piezoelectric material. The middle layer comprises two elements - a piezoelectric disk, and a ring made of non-piezoelectric material. This design protects the electrical components of the converter against harmful external factors (e.g. hightemperature, chemically active atmosphere). For such a transducer, there is an analytical model describing its deflection as a function of the applied load. Closed-form analytical equations are important tools for predicting and optimizing the operation of devices. However, it should be remembered that analytical models often use various simplifications. Such simplification, in some situations, may cause that the deflection determined with the analytical solution may differ from the actual deflection of the transducer. Therefore, in this paper the results obtained using the analytical model, were compared with the numerical results (no simplifications were used in the numerical simulation). The tests were performed for various constructional variants of the transducer, determined by selected non-dimensional parameters. These parameters include dimensions and mechanical properties of both the piezoelectric disk and passive plates. The obtained results made it possible to determine the degree of applicability of the analytical solution. It was found that the analytical model gives correct results, when the piezoelectric disk is made of various piezoelectric materials (e.g. Lead Zirconate Titanate (PZT), Polyvinylidene Fluoride (PVDF), whereas the passive components are layers made of materials used in MEMS (micro-electro mechanical system), such as silicon, silicon nitride, and most metals. Moreover, the range of relative thicknesses of individual transducer layers was determined, for which the convergence of analytical and numerical solution is obtained.
\end{abstract}

Keywords: piezoelectric transducer; electromechanical characteristic.

\section{Introduction}

Currently, piezoelectric transducers are used to measure and / or regulate a wide spectrum of physical quantities, such as force, deformation, temperature, pressure, etc. The principle of their operation is based on the conversion of electrical energy into mechanical or vice versa [1-6]. The relationship between deformation and the electric field is determined by the constitutive equation [78]. This equation is the basis for determining the electromechanical characteristics of the transducer (the relationship between deflection and the applied electrical or mechanical load). The ability to predict the conditions of transducer deformation is very useful, because it allows to increase its efficiency at the design stage. This can be accomplished, for example, by achieving greater transducer deflection when using less input power, often while reducing the transducer weight or its dimensions.f

In practice, most piezoelectric transducers usually have a layered structure, which causes some difficulties in determining their characteristics. For actual transducers, the dependence between deflection and the applied load can be determined through numerical [9-12], analytical-numerical [1215] or analytical methods. Currently, numerical methods are very popular. This is due to the fact that the majority of commercial programs supporting the design process allow relatively simple modelling of many physical processes (strength calculation [16-17], friction-flow analyses, [18-25], piezoelectricity [26]). However, many scientists, trying to provide more sophisticated design methods, develop new closed-form analytical solutions that are important tools for predicting and optimizing the operation of piezoelectric transducers. The methods of obtaining analytical solutions and their form depend mainly on the geometrical and material features of the transducers and the conditions of fastening and loading.

Analytical solutions for transducers with beam construction of equal length of the passive and piezoelectric layers are presented in papers [27-30]. In [27], a double-layer transducer was analysed. In the paper [28], there is the issue of a three-layer converter, whereas in [29-30] - a multi-layer one.

Electromechanical characteristics of two-layer transducers with various layer lengths are discussed in [31-32]. Analytical modelling of two- and three-layer transducers with freely defined boundary conditions and geometry was also discussed in [33-34]. The authors obtained the 
electromechanical characteristics of transducers using the following methods: the elementary theory of elasticity[27], the energetic method [28, 29], the Airy stress function method [30], the beam theory [31], the Lagrange method [32], the implementation of piezoelectric segments into the beam [33-34].

Membrane converters are another group of piezoelectric transducers. In works [35-38], electromechanical characteristics are given and the impact of the design parameters on the performance characteristics of two-layer piezoelectric circular transducers is discussed. The relationship between deflection and applied load was obtained using a method based on the Plate Theory. This approach can also be used for three-layer [39-40] or multi-layer [41] circular converters. Additional layers (piezoelectric or passive) in the transducer can perform various functions, e.g. they allow to increase the deflection of the transducer while reducing its dimensions [42]. The use of an additional external non-electrical layer in the transmitter design [40] can also protect the transmitter's electrical components against damage caused by external factors. Furthermore, in the event of sparking on the transducer wires / electrodes, this layer prevents possible fire.

Obtaining a closed-form analytical equation requires formulation and solution of constitutive equations. These equations combine geometric features, material properties, and physical parameters, such as, for example, the electric field. Solving such a system of equations is very difficult. The material and geometrical inhomogeneity of the global transducer structure forces the use of certain simplifications. Such simplification, in some situations, may cause that the deflection determined with the analytical solution may differ from the actual deflection of the transducer.

Therefore, in the presented work the results obtained through the analytical model [40] were compared with the numerical results (no simplifications were used in the numerical simulation). The tests were carried out for various constructional variants of the transducer, determined by selected dimensionless parameters. These parameters include dimensions and mechanical properties of both the piezoelectric disk and passive plates.

The construction of the analysed transducer, its analytical model and FEM modelling are discussed in section 2. The comparison of electromechanical characteristics obtained through the analytical and numerical solutions are given in chapter 3 . The third chapter also specifies the degree of applicability of the analytical solution. The conclusions of the study are presented in the last section.

\section{Materials and methods}

\section{Object of study}

The transducer (Fig. 1) built of four components is analysed in the work. The outer layers, $t_{1}$ and $t_{3}$ thick, are passive components.

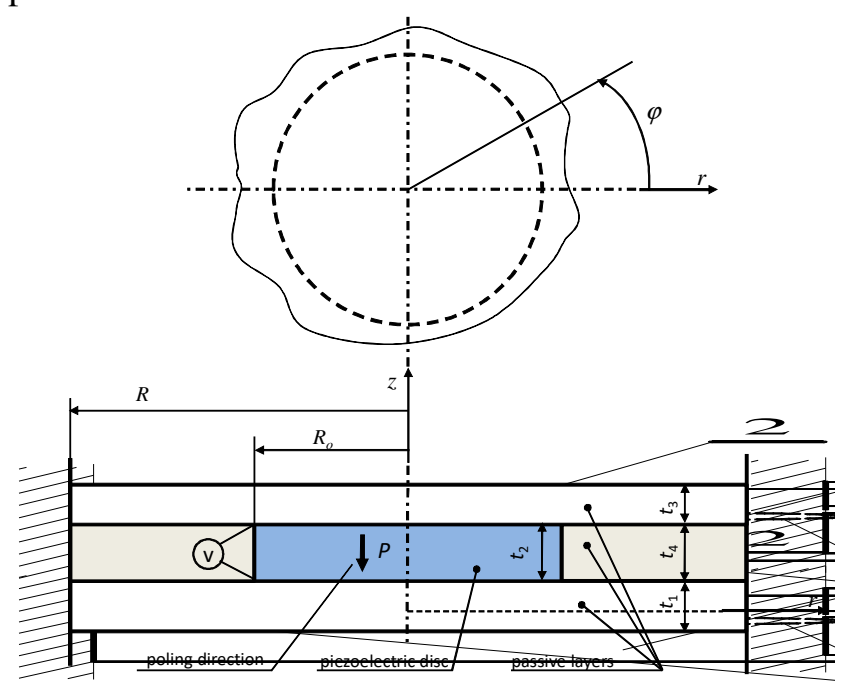

Fig. 1. Structure of the circular tree layered piezoelectric transducer

Between them there is a piezoelectric disk and a passive ring. The task of the ring, which can be made of foam, for example, is to stabilize the electrical wires that supply current to the electrodes of 
the piezoelectric disk. The disk and ring have the same thickness $-t_{2}=t_{4}$. Moreover, the outer radius of the disk is the same as the inner radius of the ring and is equal to $R_{o}$. All passive components have an identical outer radius - $R$ and are fixed on external cylindrical surfaces. Transducer deflection is caused by a transverse piezoelectric effect (caused by the action of $V$ voltage) that occurs in a piezoelectric disk. For such a transducer (Fig. 1), the authors of [40] developed an analytical model describing the relationship between deflection and the applied electrical load:

$$
w(r)=\left\{\begin{array}{l}
w_{I}(r)=\frac{A_{I}}{C}, r \leq R_{o} \\
w_{I I}(r)=\frac{A_{I I}}{C}, R_{o} \leq r \leq R
\end{array}\right.
$$

where

$$
\begin{aligned}
& A_{I}=3 d_{31} E_{2} V R_{o}^{2}\left(t_{1}+t_{2}\right)\left(1-v_{1}^{2}\right)^{2}\left(1+v_{2}\right)\left(r^{2}\left(1-\frac{R^{2}}{R_{o}^{2}}\right)+2 R^{2} \ln \left(\frac{R}{R_{o}}\right)\right), \\
& A_{I I}=3 d_{31} E_{2} V R_{o}^{2}\left(t_{1}+t_{2}\right)\left(1-v_{1}^{2}\right)^{2}\left(1+v_{2}\right)\left(\left(r^{2}-R^{2}\right)+2 R^{2} \ln \left(\frac{R}{r}\right)\right), \\
& C=\left(1-v_{1}^{2}\right)\left(v_{2}^{2}-1\right)\left(2 E_{1} R^{2} t_{1}^{3}+\frac{\left(v_{1}^{2}-1\right)\left(F_{I}+\left(-1+v_{2}\right)\left(2 E_{3} t_{3}\left(F_{I V}+F_{I I}\right)\left(-1+v_{4}{ }^{2}\right)+F_{I I I}\right)\right)}{\left(v_{2}-1\right)\left(v_{3}^{2}-1\right)\left(v_{4}{ }^{2}-1\right)}\right), \\
& F_{I}=E_{2}\left(v_{3}^{2}-1\right)\left(v_{4}^{2}-1\right)\left(R^{2}-R o^{2}\right)\left(3 t_{1}^{2}+6 t_{1} t_{2}+4 t_{2}{ }^{2}\right) t_{2}, \\
& F_{I I}=R^{2}\left(3 t_{1}^{2}+6 t_{1} t_{2}+6 t_{2}{ }^{2}+6 t_{1} t_{3}+6 t_{2} t_{3}+4 t_{3}{ }^{2}+6 t_{1} t_{4}+6 t_{3} t_{4}+6 t_{4}{ }^{2}+6\left(t_{2}-t_{4}\right)\left(t_{1}+t_{2}+t_{3}+t_{4}\right) v_{3}\right), \\
& F_{I I I}=E_{4} t_{4}\left(3 t_{1}^{2}+6 t_{1} t_{4}+4 t_{4}{ }^{2}\right)\left(v_{3}^{2}-1\right)\left(R_{o}{ }^{2}\left(v_{4}+1\right)-R^{2}\left(v_{4}-1\right)\right), \\
& F_{I V}=-6 R_{o}^{2}\left(t_{2}-t_{4}\right)\left(t_{1}+t_{2}+t_{3}+t_{4}\right)\left(1+v_{3}\right), \\
& E_{i}-\text { Young's modules, } v_{i} \text {-Poisson's ratios, } d_{31} \text {-piezoelectric constant. }
\end{aligned}
$$

The analytical model was developed using the constitutive compounds of piezoelectric materials and Plate Theories [43]. The analytical model has been positively verified. However, the approach used by the authors of the paper [40] enforces the use of certain simplifications, such as, e.g. skipping shear deformation and neglecting the longitudinal and shear piezoelectric effects. Studies on doublelayer round transducers [44- 45] have shown that such simplifications (for certain geometrical-material configurations of transducers) cause significant errors in predicting transducer deflection using the analytical model. Therefore, the need to determine the scope of validity of the analytical solution is justified (1). This scope can be determined, e.g. by comparing the results obtained from the analytical model with the results obtained using numerical methods (without using any simplifications in the numerical models). Therefore, for various geometrical and material variants of the transducer (determined by selected non-dimensional variables), its electromechanical characteristics were determined (analytically and numerically). Analytical solutions were obtained on the basis of equation (1). As for the numerical tests, they were performed using the finite element method (FEM). FEM modelling is discussed in the next subsection.

\section{2. $\quad$ FEM modelling}

Numerical simulations were performed using COMSOL Multiphysics software [46-48]. Figure 2 shows, for an arbitrarily chosen geometrical configuration of the transducer, division into finite elements and applied boundary conditions. A half symmetry axisymmetric model of the transducer was built using 6-node triangular plane elements. A self-adaptive finite element mesh was used, matching the modelled geometry and physical phenomenon (in COMSOL environment this option is called "Physics-controlled mesh"). In addition, the mesh was manually condensed at the end of both the piezoelectric disk and the transducer. 


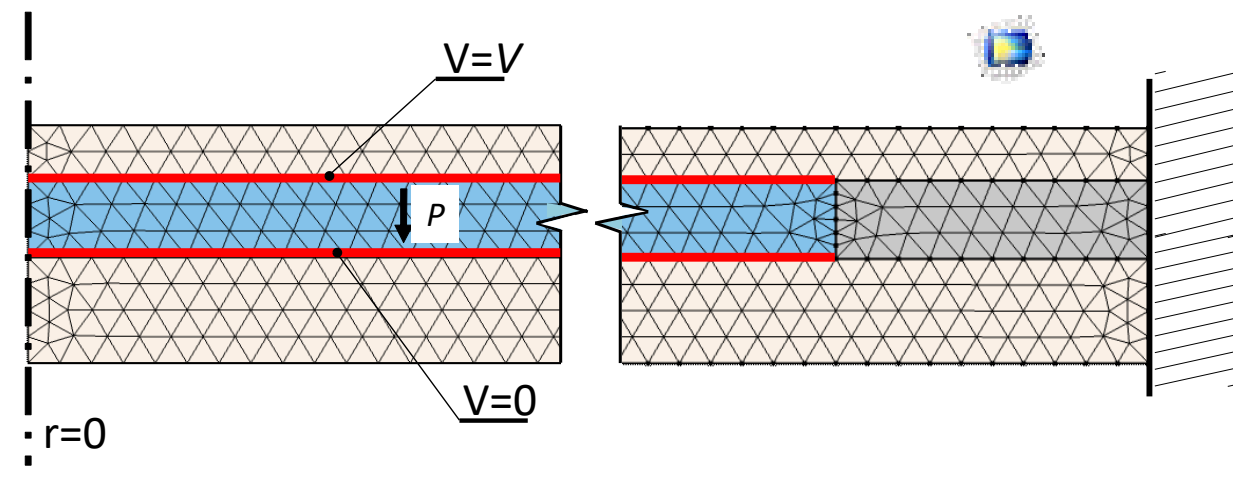

Fig. 2. Finite element mesh and boundary conditions

It is worth mentioning that when modelling piezoelectric materials using COMSOL software, there is no need to choose manually specific type of element. The finite element type is selected automatically depending on the modelled physical problem.

As for the boundary conditions, the transducer outer cylindrical surfaces are fixed supported and the electrical load $(V=0$ and $V=100 \mathrm{~V})$ is applied to the nodes lying in the connection planes of the piezoelectric disk with the passive layers (red lines in Figure 2).

Numerical simulations were performed for various constructional and material variants of the transducer. In the analyses performed, it was assumed that the external radius of the transducer is constant and equals: $R=6 \times 10^{-2} \mathrm{~m}$. The thickness $\left(t_{2}=t_{4}=5 \times 10^{-4} \mathrm{~m}\right)$ and material of components in the middle layer are also constant. The piezoelectric disk is made of PZT-5H (Lead Zirconate Titanate), while the middle ring is made of foam. The properties of these materials are shown in the table below.

Table 1

Properties of PZT-5H and foam

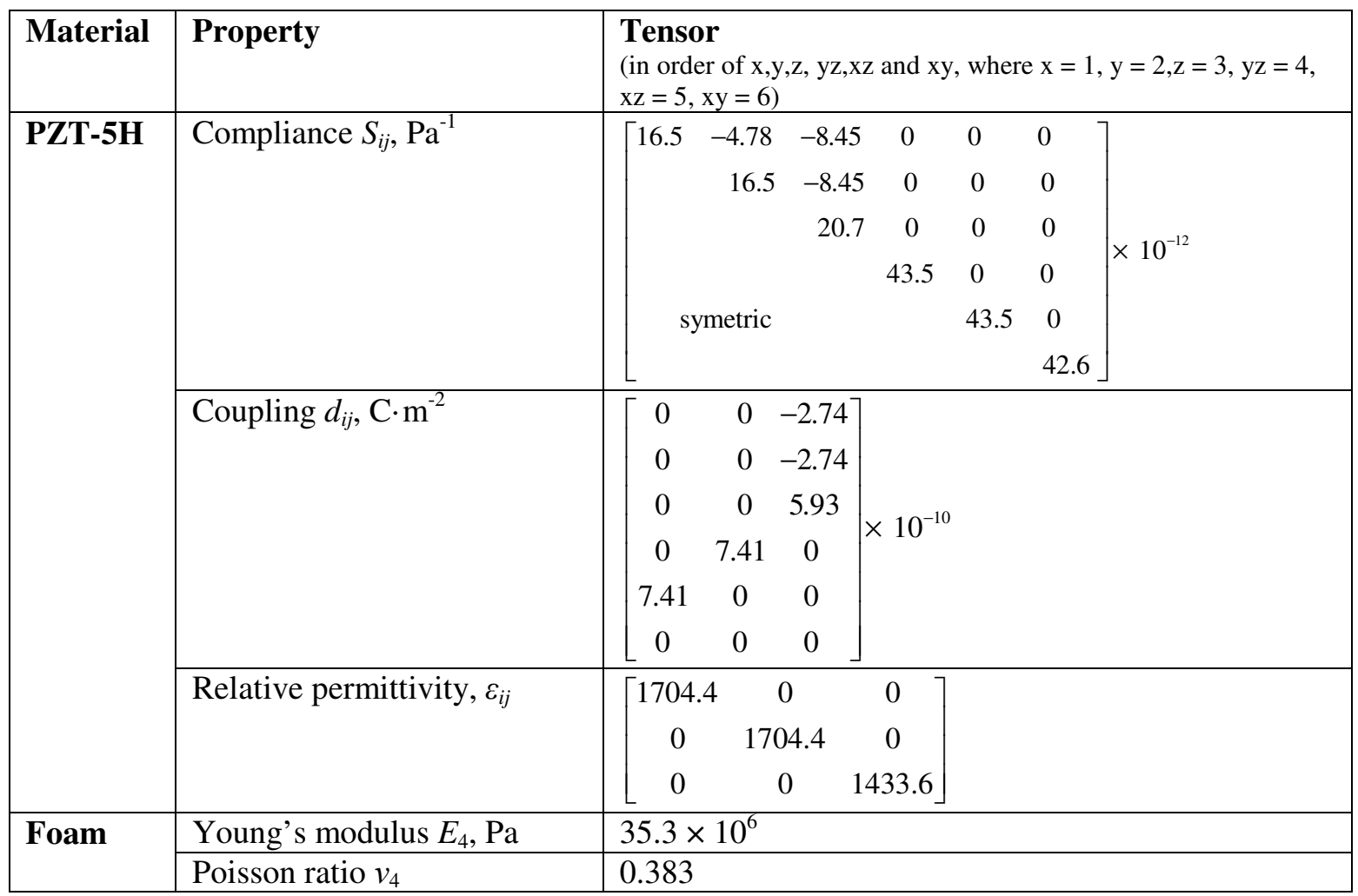

As for the outer layers, it was assumed that both layers have the same Poisson's ratio, which is $v_{1}=v_{3}=.0 .33$. Young's modules $\left(E_{1}, E_{3}\right)$ and thicknesses $\left(t_{1}, t_{3}\right)$ of these layers were variable. Furthermore, the calculations were performed for various values of the radius of the piezoelectric disk 
$R_{o}$. The range of variability of the above parameters (defined by non-dimensional variables) is discussed in the next chapter. As regards the applied electrical load, it was always $V=100 \mathrm{~V}$.

\section{Results and discussion}

As already mentioned, the goal of this work was to determine the scope of applicability of the analytical model of the analysed transducer. The numerical solutions developed were not used to assess the accuracy of the analytical solution. FEM solutions (without simplifications, which were assumed in the exact solution) were used for comparative analyses. By comparing the analytical and numerical solutions, it was possible to determine the range of transducer parameters for which both solutions coincide (parameters determine the range in which, despite simplifications, an analytical solution is suitable).

When determining the degree of applicability, consideration should be given to both the geometrical and material features of the transducer. Therefore, analyses were made for the following non-dimensional variables (including variability of geometry $\left(t_{g}, R_{g}\right)$ and transducer materials $\left(E_{\mathrm{g}}\right)$ :

- the relative thickness of piezo and non-piezo elements: $t_{g}=t_{2} /\left(t_{1}+t_{3}\right)$;

- the relative radius of the piezoelectric disk and non-piezoelectric layers: $R_{g}=R_{o} / R$;

- the elastic moduli ratio of piezo and non-piezoelectric components: $E_{g}=E_{2} /\left(E_{1}+E_{3}\right)$.

For the arbitrarily assumed range of variation of the tan parameters $R_{g}$ and $E_{\mathrm{g}}$, the deflection of the transducer centre point $(r=0)$ was calculated using the formula (1). The results obtained in this way were compared with the FEM results. In the analytical solution only the transverse piezoelectric effect was taken into account $\left(d_{31}=-2.74 \cdot 10^{-10} \mathrm{C} / \mathrm{N}\right)$. Furthermore, in the analytical model it was assumed that the piezoelectric material is isotropic, and its Young's modulus and Poisson's ratio are respectively: $\quad E_{2}=1 / S_{11}=6.06 \cdot 1010 \mathrm{~Pa}, \quad v_{2}=-S_{12} / S_{11}=0.289$. Other geometrical and material parameters used in the analytical solution were the same as in the numerical models.

a)

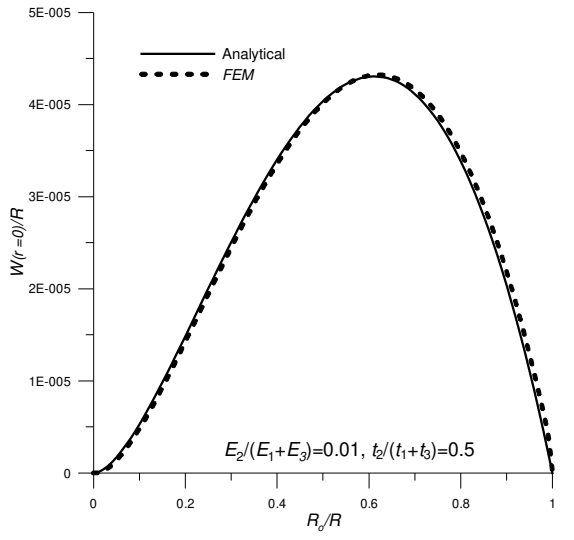

b)

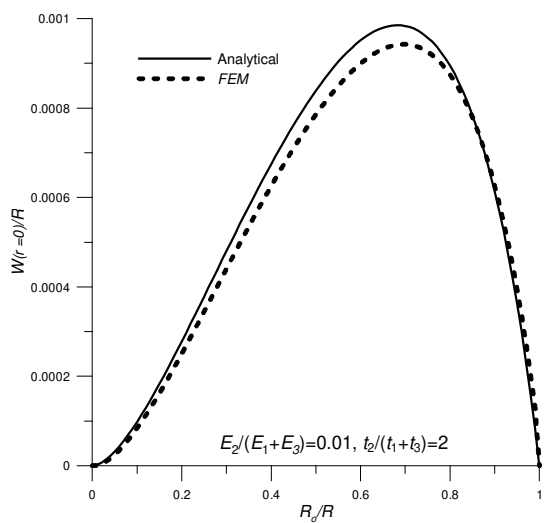

c)

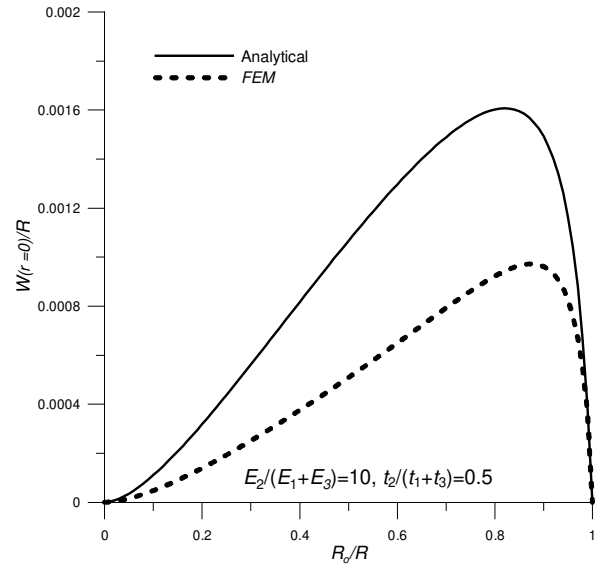

Fig. 3. Transducer deflection in the $\boldsymbol{R}_{g}$ parameter domain: a $-E_{g}=0.01, t_{g}=0.5$;

$$
\mathrm{b}-E_{g}=0.01, t_{g}=2 ; \mathrm{c}-E_{g}=10, t_{g}=0.5
$$


An important thing in predicting the transducer behaviour is to determine the optimal ratio of the piezoelectric disk radius and passive layers $-R_{g}$. Therefore, in the first stage of the research, characteristics describing transducer deflection in the domain of the $R_{g}$ parameter were developed. The obtained characteristics, determined for various values of parameters $t_{g}$ and $E_{\mathrm{g}}$, are shown in Figure 3 .

Analysing the obtained results, it can be stated that the optimal values of the $R_{g}$ parameter (values for which the highest deflection of the transducer is obtained), determined using FEM and analytical solutions, are almost identical for small $E_{g}$ values and similar, when using components with a greater diversity of Young's moduli and relative layer thickness (for $E_{g}=10$ the error is about $5.8 \%$ ). Furthermore, as the value of $E_{g}$ and $t_{g}$ parameters increases, the error in the determined transducer deflection increases - the deflection determined through the analytical solution is greater than the deflection obtained from numerical simulations. Therefore, it was necessary to develop the electromechanical characteristics of the transducer in the domain of $E_{g}$ and $t_{g}$.

a)

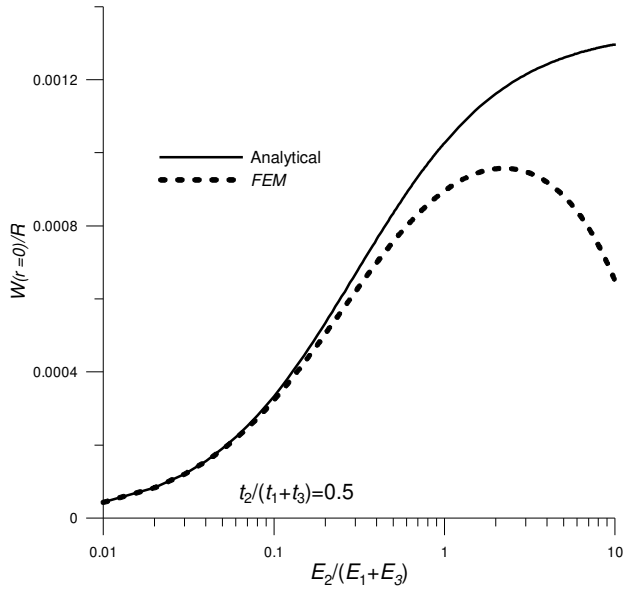

b)

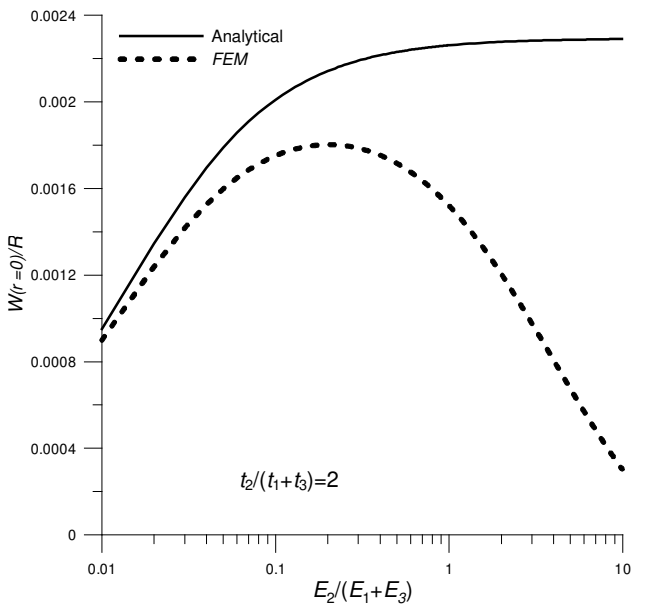

Fig. 4. Transducer deflection in the $\boldsymbol{E}_{g}$ parameter domain $\left(\boldsymbol{R}_{g}=\mathbf{0 6}\right)$, a- $t_{g}=0.5$, b- $t_{g}=2$

Table 2

$E_{g}$ parameter values for associated materials used in piezoelectric transducers, Young's moduli ( $\boldsymbol{E}_{2}$ - for passive materials, $\boldsymbol{E}_{1,3}$ - piezolectric materials) is given in brackets

\begin{tabular}{|c|c|c|c|c|c|c|}
\hline & \multicolumn{5}{|c|}{ Piezoelectric materials } & \\
\hline & $\begin{array}{l}\text { soft APC } \\
(66.6 G P a)\end{array}$ & $\begin{array}{c}\text { soft PTZ } \\
(\mathbf{6 2 . 1 G P a )}\end{array}$ & $\begin{array}{c}\text { PVDF } \\
\text { (3 GPa) }\end{array}$ & $\begin{array}{l}\text { hard APC } \\
(\mathbf{8 5 . 4} \mathbf{G P a})\end{array}$ & $\begin{array}{l}\text { hard PTZ } \\
(\mathbf{8 6 . 9 G P a )}\end{array}$ & \\
\hline \multirow{8}{*}{ 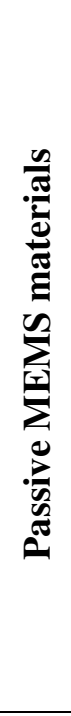 } & $\begin{array}{l}\text { Silicon } \\
\text { (129 GPa) }\end{array}$ & 0.26 & 0.24 & 0.012 & 0.33 & 0.34 \\
\hline & $\begin{array}{l}\text { Silicon Oxide } \\
\text { (73 GPa) }\end{array}$ & 0.46 & 0.43 & 0.021 & 0.58 & 0.60 \\
\hline & $\begin{array}{l}\text { Silicon nitride } \\
\text { (304 GPa) }\end{array}$ & 0.11 & 0.10 & 0.005 & 0.14 & 0.14 \\
\hline & $\begin{array}{l}\text { Silicon Carbide } \\
\text { (430 GPa) }\end{array}$ & 0.08 & 0.07 & 0.003 & 0.10 & 0.10 \\
\hline & $\begin{array}{l}\text { Nickel } \\
\text { (207 GPa) }\end{array}$ & 0.16 & 0.15 & 0.007 & 0.21 & 0.21 \\
\hline & $\begin{array}{l}\text { Aluminum } \\
(69 \mathrm{GPa})\end{array}$ & 0.48 & 0.45 & 0.022 & 0.62 & 0.63 \\
\hline & $\begin{array}{l}\text { Aluminum Oxide } \\
\text { (393 GPa) }\end{array}$ & 0.08 & 0.08 & 0.004 & 0.11 & 0.11 \\
\hline & $\begin{array}{l}\text { Copper } \\
\text { (135 GPa) }\end{array}$ & 0.25 & 0.23 & 0.011 & 0.32 & 0.32 \\
\hline
\end{tabular}

These characteristics made it possible to determine the limit values of dimensionless variables, beyond which a divergence of analytical and numerical solutions occurs. The characteristics in the domain of $E_{g}$ are shown in Figure 4. 
Analysing the characteristics presented in Figure 4, it can be seen that the limit value of the $E_{g}$ parameter, after exceeding which there is a divergence of both solutions (the assumption of linearity of deformations in the analytical solution loses validity), strongly depends on the relative thickness of the layers in the transducer. Therefore, for typical materials used for passive [49] and piezoelectric [50] elements in MEMS devices, $E_{g}$ values were calculated (assuming that the outer layers are made of the same material $E_{1}=E_{3}$ ), as shown in Table 2. Then, for selected specific parameters $E_{g}$ (Table 2), electromechanical characteristics in the domain of the $t_{g}$ parameter were determined. The obtained characteristics are shown in Figure 5.

a)

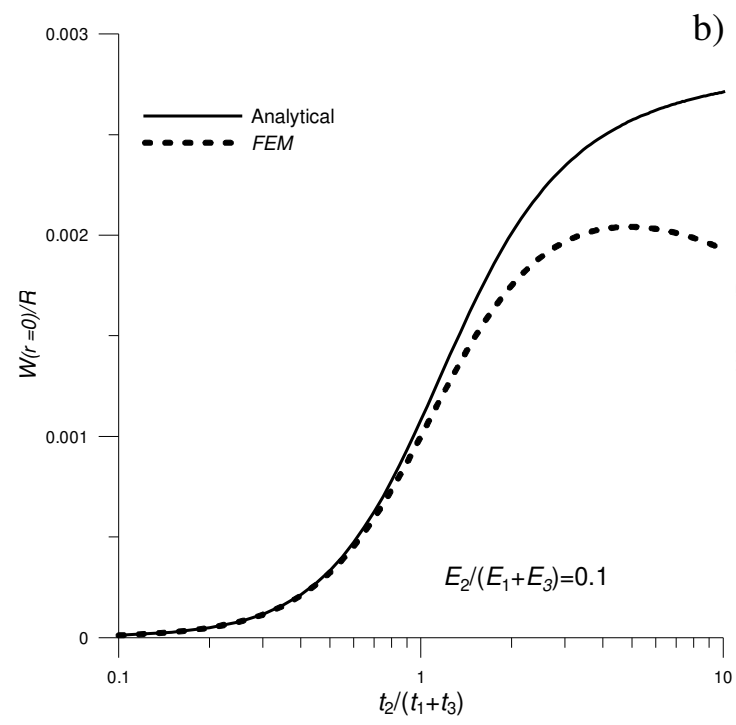

b)

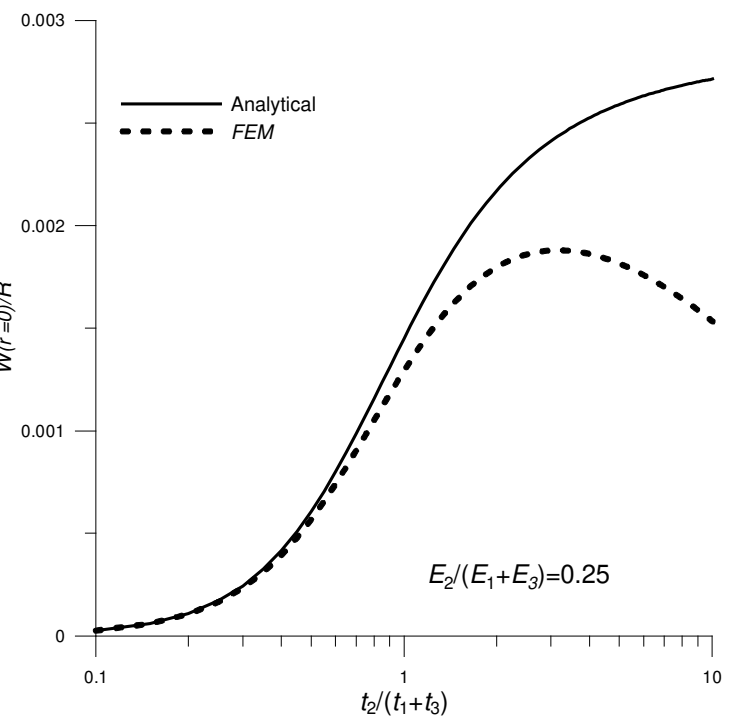

c)

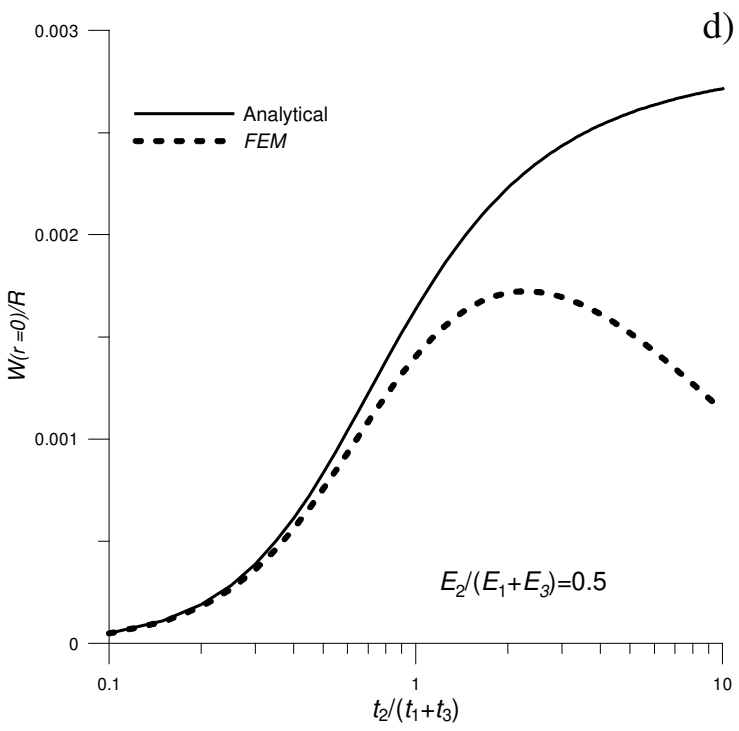

d)

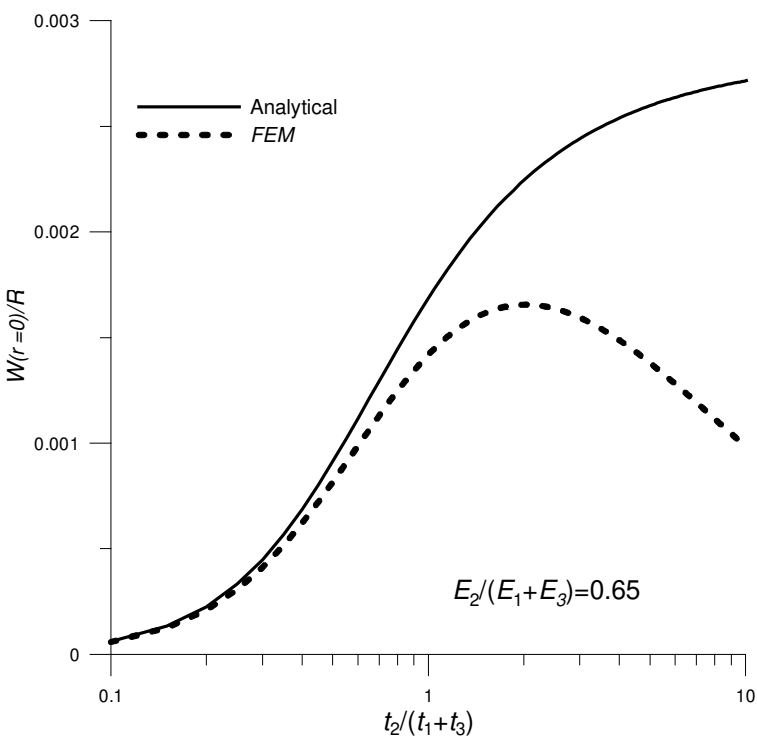

Fig. 5. Transducer deflection in the tg parameter domain $\left(\boldsymbol{R}_{\boldsymbol{g}}=\mathbf{0 . 6}\right): \mathrm{a}-E_{g}=0.1$;

$$
\mathrm{b}-E_{g}=0.25 ; \mathrm{c}-E_{g}=0.5 ; \mathrm{d}-E_{g}=0.25
$$

Analysing the obtained results, it can be stated that for piezoelectric transducers made of typical materials used in MEMS, convergence of analytical and numerical solutions is always obtained, when the $t_{g}$ parameter is less than 1 (in most commonly used transducers $t_{g}<1.5$ ). What is more, the error between the two solutions increases, as the relative thickness varies. It should also be noted that after exceeding a certain value of the $t_{g}$ parameter (e.g. $t_{g}=2.8$, Fig. $5 \mathrm{c}$ ), the assumption about the linearity of deformation of individual transducer components adopted in the analytical solution loses validity. Therefore, in such situation the analytical model should not be used in predicting the transducer performance. 


\section{Conclusions}

1. Based on the results obtained, it can be concluded that the analytical solution can always be used to predict the optimal relative radius of the piezoelectric disk and non-piezoelectric layers (minimal (for $E_{g}=0.01$ ) and maximal (for $E_{g}=10$ ) errors are $0.8 \%$ and $5.8 \%$ accordingly).

2. The analysis shows that the analytical model can be used for predicting the transducer deflection value, when the piezoelectric disk is made of various piezoelectric materials (e.g. PZT, PVDF, APC), and the passive components from materials commonly used in MEMS, such as silicon, silicon nitride, silicon oxide and most metals.

3. However, it should be remembered that the accuracy of the results obtained depends on the relative thickness of the transducer layers (in general, the results obtained from the analytical solution are sensible, when the $t_{g}$ parameter is less than 1.5- after exceeding this threshold, the solutions are divergent and the error is greater than $15 \%$ ).

\section{Funding}

This publication was financed through the program of the Ministry of Science and Higher Education of Poland named "Regional Initiative of Excellence" in 2019-2022 project number 011/RID/2018/19.

\section{Acknowledgements}

This research was co-founded through the subsidy of the Ministry of Science and Higher Education for the discipline of Mechanical Engineering at the Faculty of Mechanical Engineering, Bialystok University of Technology.

\section{References}

[1] Busch-Vishniac I.J. Electromechanical Sensors and Actuators, New York: Springer, 1999. 338 p.

[2] Ştefănescu D.M. Handbook of Force Transducers: Principles and Components, Berlin/Heidelberg: Springer, $2011.590 \mathrm{p}$.

[3] Caliano G., N. Lamberti G., Iula A., Pappalardo M., A piezoelectric bimorph static pressure sensor. Sensors and Actuators A, vol. 46, 1995, pp. 176-178.

[4] Levinzon F. Piezoelectric Accelerometers with Integral Electronics. Berlin/Heidelberg: Springer, 2015. 169 p.

[5] DeVoe D.L. Piezoelectric thin film micromechanical beam resonators. Sensors and Actuators A, vol. 88, 2001, pp. 263-272.

[6] Tzou H.S. Piezoelectric Shells: Distributed Sensing and Control of Continua, Dordrecht: Kluwer Academic Publishers, 1999, 456 p.

[7] Curie P.J., Curie J., Crystal Physics-Development by Pressure 0/ Polar Electricity in Hemihedral Crystals with Inclined Faces, Proceedings of the Academy of sciences(Paris), vol. 91, 1880. 294 p. (In French)

[8] Berlincourt D. A., Curran D. R., Jaffe H., Mason W. P., Piezoelectric and Piezomagnetical Materials and Their Function in Transducers. In: Mason, W.P., Ed., Physical Acoustics, New York: Academic Press, 1A, 1964, pp. 169-270.

[9] Rahmoune, M.; Osmont, D. Classic finite elements for simulation of piezoelectric smart structures. Mechanika, vol. 86, 2010, pp. 50-57.

[10] Lašová Z., Zemčík R. Comparison of Finite Element Models for Piezoelectric Materials. Procedia Engineering, vol. 48, 2012, pp. 375-380

[11] Shah A. A. A FEM-BEM interactive coupling for modeling the piezoelectric health monitoring systems. Latin American Journal of Solids and Structures, vol.8(3), 2011, pp. 305-334.

[12] Dobrucki A.B., Pruchnicki P. Theory of piezoelectric axisymmetric bimorph. Sensors and Actuators A, vol. 58, 1997, pp. 203-212.

[13] Yang J., Yang Z. Analytical and numerical modeling of resonant piezoelectric devices in China-A review. Science in China Series G Physics Mechanics and Astronomy, vol. 51, 2008, pp. 17751807.

[14]Bowen C., Perry A., Stevens R., Mahon S. Analytical and numerical modelling of 3-3 piezoelectric composites. Integrated Ferroelectrics, vol. 32, 2001, pp. 333-342. 
[15] Imperiale S., Joly P. Mathematical and numerical modelling of piezoelectric sensors. ESAIM: Mathematical Modelling and Numerical Analysis, EDP Sciences, 2012. ffhal-00869631f.

[16] Rogowski G. Plastic zones for 3D planar cracks embedded in an elastic-plastic layer sandwiched between two elastic adherents. Theoretical and Applied Fracture Mechanics, vol. 98, 2018, pp. 199-209.

[17] Mieczkowski G., Molski K., Seweryn A. Finite-element modeling of stresses and displacements near the tips of pointed inclusions. Materials Science, vol. 43( 2), 2007, pp. 183-194.

[18] Łukaszewicz A. Temperature field in the contact zone in the course of rotary friction welding of metals, Materials Science, vol. 55 (1), 2019, pp. 39-45.

[19] Trochimczuk R., Łukaszewicz A., Mikołajczyk T., Aggogeri F., Borboni A. Finite element method stiffness analysis of a novel telemanipulator for minimally invasive surgery. SIMULATION, vol. 95, 2019, pp. 1015-1025.

[20] Szpica D. Investigating fuel dosage non-repeatability of low pressure gas-phase injectors. Flow Measurement and Instrumentation, vol. 59, 2018, pp. 147-156.

[21] Szpica D. Simplified numerical simulation as the base for throttle flow characteristics designation. Mechanika, vol. 21(2), pp. 129-133;

[22] Szpica D. Modeling of the operation of a Dual Mass Flywheel (DMF) for different engine-related distortions. Mathematical and Computer Modelling of Dynamical Systems, vol. 24(6), 2018, pp. 643-660.

[23] Borawski A. Suggested Research Method for Testing Selected Tribological Properties of Friction Components in Vehicle Braking Systems. Acta Mechanica et Automatica, vol. 10(3), 2016, pp. 223-226.

[24] Borawski A. Common methods in analysing the tribological properties of brake pads and discs - a review. Acta Mechanica et Automatica, vol. 13(3), 2019, pp. 189-199.

[25] Borawski A. Simulation Study of the Process of Friction in the Working Elements of a Car Braking System at Different Degrees of Wear. Acta Mechanica et Automatica, vol. 12(3), 2018, pp. 221-226.

[26] Nguyen V.T., Kumar P., Leong J.Y.C. Finite Element Modelling and Simulations of Piezoelectric Actuators Responses with Uncertainty Quantification. Computation 2018, vol. 6(4), 60.

[27] Chen N. Yan P., Ouyang J. A generalized approach on bending and stress analysis of beams with piezoelectric material bonded. Sensors and Actuators A, vol. 290, 2019, pp. 54-61.

[28] Wang Q.M., Cross L.E. Constitutive equations of symmetrical triple layer piezoelectric benders. IEEE Transactions on Ultrasonics, Ferroelectrics and Frequency Control, vol. 46, 1999, pp.13431351.

[29]Ballas R.G., Schlaak H.F., Schmid A.J. The constituent equations of piezoelectric multilayer bending actuators in closed analytical form and experimental results. Sens. Sensors and Actuators A, vol.130-131, 2006, pp. 91-98.

[30]Xiang H.J., Shi Z.F. Static analysis for multi-layered piezoelectric cantilevers. International Journal of Solids and Structures, vol. 45, pp. 113-128.

[31] Park J.K., Moon W.K. Constitutive relations for piezoelectric benders under various boundary conditions. Sensors and Actuators A, vol. 117, 2005, pp. 159-167.

[32] Raeisifard H., Bahrami M.N., Yousefi-Koma A., Fard H.R. Static characterization and pull-in voltage of a micro-switch under both electrostatic and piezoelectric excitations. European Journal of Mechanics, vol. 44, 2014, pp. 116-124.

[33] Mieczkowski G. Electromechanical characteristics of piezoelectric converters with freely defined boundary conditions and geometry. Mechanika, vol. 22(4), 2016, pp. 265-272.

[34] Mieczkowski G. The constituent equations of piezoelectric cantilevered three-layer actuators with various external loads and geometry. Journal of Theoretical and Applied Mechanics, vol. 55(1), 2017, pp. 69-86.

[35] Adelman N.T., Stavsky Y. Flexural-extensional behavior of composite piezoelectric circular plates. Journal of the Acoustical Society of America, vol. 67, 1980, pp. 819-822.

[36] Yanagisawa T., Nakagawa Y. Determination of Optimum Dimensions for Unimorph Type PiezoElectric Loudspeaker. Transactions of the Institute of Electronics, Information and Communication Engineers A, vol. J76, 1993, pp. 1261-1269. 
[37] Mo C., Wright R., Slaughter W.S., Clark W.W. Behaviour of a unimorph circular piezoelectric actuator. Smart Materials and Structures, vol. 15, 2006, pp. 1094-1102.

[38] Guan E., Ge Y., Liu J., Yan W., Zhao Y. Piezoelectric Micro-Pump Suction Cup Design and Research on the Optimal Static Driving Characteristics. Proceedings of the Intelligent Robotics and Applications, August 16-18, 2017, Cham, China, pp. 26-38.

[39] Yihua H., Wenjin H. Research on the displacement function and equivalent circuit of circular flexural vibration mode piezoelectric ceramic composite transducers. IEEE transactions on ultrasonics, ferroelectrics, and frequency control, vol. 60, 2013, pp. 218-234.

[40] Mieczkowski G., Borawski A., Szpica D. Static electromechanical characteristic of a three-layer circular piezoelectric transducer, Sensors, vol. 20, 2020, 222, 14p

[41] Li X., Du H., Xu L., Hu Y., Xu L. Optimization of a circular thin-film piezoelectric actuator lying on a clamped multilayered elastic plate. IEEE transactions on ultrasonics, ferroelectrics, and frequency control, vol. 56, 2009, pp. 1469-1475.

[42] Dong S., Du X.-H., Bouchilloux P., Uchino K. Piezoelectric Ring-Morph Actuators for Valve Application. Journal of Electroceramics, vol. 8, 2002, pp. 155-161.

[43] Timoshenko, S.; Woinowsky-Krieger, S. Theory of Plates and Shells. Second edition. New York: McGraw-Hill College, 1959. 575 p.

[44]Li S., Chen S. Analytical analysis of a circular PZT actuator for valveless micropumps. Sensors and Actuators A, vol. 104, 2003, pp. 151-161.

[45]Cao L., Mantell S., Polla D. Design and simulation of an implantable medical drug delivery system using microelectromechanical systems technology. Sensors and Actuators A, vol. 94, 2001, pp. 117-125.

[46] Structural Mechanics Module, COMSOL Doc.. [online] [30. 11. 2019]. Available at: https://doc.comsol.com/5.5/docserver/\#!TOC:/com.comsol.help.sme/toc.xml:SOURCE:resource_t ocfile_-1058655296.html.

[47]MEMS Module, COMSOL Doc. [online] [30. 11. 2019]. Available at: https://doc.comsol.com/5.5/docserver/\#!TOC:/com.comsol.help.mems/toc.xml:SOURCE:resource _tocfile_573990530.html.

[48] Piezoelectric Shear-Actuated Beam COMSOL Doc. [online] [30. 11. 2019]. Available at: https://www.comsol.com/model/piezoelectric-shear-actuated-beam-24.

[49] Spearing S. M. Materials issues in microelectromechanical systems (MEMS). Acta Materialia, vol.48(1), 2000, pp. 179-196.

[50] Tichý J., Erhart J., Kittinger E., Prívratská J. Fundamentals of Piezoelectric Sensorics Mechanical, Dielectric, and Thermodynamical. Berlin/Heidelberg: Springer, 2010. 207 p. 